\title{
热电器件的界面和界面材料
}

\author{
胡晓凯 ${ }^{1,4}$, 张双猛 ${ }^{1}$, 赵 府 ${ }^{1,2}$, 刘 勇 ${ }^{1,3}$, 刘玮书 ${ }^{1}$
}

(1. 南方科技大学 材料科学与工程系, 深圳 518055 ; 2. 南方科技大学 前沿与交叉科学研究院, 深圳 518055; 3. 中 国航发北京航空材料研究院, 北京 100095; 4. 迪肯大学 前沿材料研究所, 吉郎 3216, 澳大利亚)

摘 要: 基于塞贝克效应的热电转换技术, 在大量分散的低品位废热转换电能方面有着不可替代的优势。以热电优 值 $Z T$ 为性能指标的热电材料研发成为新能源材料领域研究的热点之一。近年来, 大量新型中温热电材料被相继发 现, 然而新型热电材料的产业化应用, 尤其是在温差发电方面的进展尤为缓慢, 其中热电器件中的材料界面问题 严重制约了热电转换技术的应用进程。本文从 $\mathrm{Bi}_{2} \mathrm{Te}_{3}$ 型器件在温差发电方面所遇到的技术瓶颈为例, 阐述热电器 件中的界面关键技术, 并归纳出电极接触界面需要综合考虑低的界面电阻、高的结合强度、以及好的高温稳定性能。 然后总结了与 $\mathrm{Bi}_{2} \mathrm{Te}_{3} 、 \mathrm{PbTe} 、 \mathrm{CoSb}_{3}$ 基三种热电材料相关的界面材料研究进展。

关＼cjkstart键＼cjkstart词：热电器件；金属化层；界面电阻；高温稳定；综述

中图分类号: TN37 文献标识码: A

\section{Thermoelectric Device: Contact Interface and Interface Materials}

\author{
HU Xiao-Kai ${ }^{1,4}$, ZHANG Shuang-Meng $^{1}$, ZHAO Fu $^{1,2}$, LIU Yong ${ }^{1,3}$, LIU Wei-Shu ${ }^{1}$
}

(1. Department of Material Science and Engineering, Southern University of Science and Technology, Shenzhen 518055, China; 2. Academy for Advanced Interdisciplinary Studies, Southern University of Science and Technology, Shenzhen 518055, China; 3. AECC Beijing Institute of Aeronautical Materials, Beijing 100095, China; 4. Institute for Frontier Materials, Deakin University, Geelong 3216, Australia)

\begin{abstract}
Thermoelectric power generation via Seebeck effect features an unique advantage in converting large amount of distributed and low-grade waste heat into electricity. Thermoelectric materials have become a hot topic of research in the field of new energy materials, guided by the high figure of merit $Z T$. Although various mid-temperature thermoelectric materials were discovered, the industrial application of these materials, especially in power generation applications, progressed very slowly. The staggering interface technology associated with thermoelectric device restricted the advance of thermoelectric conversion technology. In this review, the bottleneck issues of utilizing $\mathrm{Bi}_{2} \mathrm{Te}_{3}$-based devices for power generation were used as an example to illustrate the critical interface technologies. The key issues at designing electrode contact interfaces were summarized, including low contact resistance, high bonding strength, and superior thermal chemical stability at high temperature. The recent progress on the metallization and interfacial barrier layer for typical materials of $\mathrm{Bi}_{2} \mathrm{Te}_{3}, \mathrm{PbTe}$ and $\mathrm{CoSb}_{3}$ were also reviewed.
\end{abstract}

Key words: thermoelectric device; metallization; contact resistance; high-temperature stability; review

随着世界经济快速发展，全球能源消耗呈爆发式增长，非可再生能源储量正以每年 150 亿吨标准

收稿日期: 2018-06-21; 收到修改稿日期：2018-08-23

基金项目: “千人计划”-青年人才项目; 深圳市“孔雀人才计划”; 广东省“珠江人才计划”-创新创业团队项目(2016ZT06G587) National 1000-Youth Talent; Shenzhen Talent Peacock Plan; Guangdong Innovative and Entrepreneurial Research Team Program (2016ZT06G587)

作者简介: 胡晓凯(1978-), 男, 博士. E-mail: xiaokai.hu@deakin.edu.au

通讯作者: 刘玮书, 副教授. E-mail: liuws@sustc.edu.cn 
煤的速度减少, 能源危机已演变为世界各国的能源 战略问题。然而, 全世界以各种形式获取的能量中 有近 $60 \%$ 以废热形式损失掉。以深圳大亚湾核电站 为例, 每年以废热形式损失掉的核能超过 300 亿千 瓦时。在交通运输行业, 机动车所消耗的汽油中, 同 样有超过 $50 \%$ 的能量以废热形式浪费掉。随着我国 人民生活水平的提高, 能耗需求也在逐步增加, 依 据中国节能协会发布的数据 ${ }^{[1]}$, 城镇居建电力消费 强度与人均 GDP 呈明显的线性关系, 人均 GDP 每增 加 1 万元, 每平米居住建筑电力消费约增加 $3 \mathrm{kWh}$ 。

2015 年, 中国建筑能源消费总量为 8.57 亿吨标准煤, 占全国能源消费总量的 $20 \%$ 。热电转换技术由于无 运动部件、无排放、结构简单, 在将大量分散的低 品位废热转换为电能方面有着不可替代的优势。

热电转换器件 (也称温差发电器件) 是一种以电 子和声子作为能量转换载体的热机, 在给定热端温 度 $T_{\mathrm{h}}$ 和冷端温度 $T_{\mathrm{c}}$ 的边界条件下, 其最大的能量转 换效率为 ${ }^{[2]}$ :

$$
\eta_{\max }=\frac{T_{\mathrm{h}}-T_{\mathrm{c}}}{T_{\mathrm{h}}} \frac{\sqrt{1+Z T_{\mathrm{ave}}}-1}{\sqrt{1+Z T_{\mathrm{ave}}}+T_{\mathrm{c}} / T_{\mathrm{h}}}
$$

其能量转换效率与卡诺效率和无量纲的材料性能优 值 $Z T$ 相关 $\left(Z T=T S^{2} \sigma /\left(\kappa_{\mathrm{carr}}+\kappa_{\mathrm{lat}}\right)\right.$, 其中 $T 、 S 、 \sigma 、 \kappa_{\mathrm{carr}}$ 和 $\kappa_{\text {lat }}$ 分别是温度、塞贝克系数、电导率、电子热 导率和晶格热导率), 热电优值 $Z T$ 为衡量热电材料 的性能指标。自上世纪末, 热电材料研究迎来新的 春天, “结构纳米化” 和“新材料”成为热电材料研究 关注的重点 ${ }^{[3-4]}$ 。随后, 研究相继发现大量新型中温 热电材料, 比如填充方钴矿化合物、复杂笼状化合 物、Zintl 相化合物、层状氧化物、离子液体化合物、 膺立方化合物等, 以及纳米材料合成工艺也被应用 于纳米热电材料的制备与研究, 比如: 高能球磨、 熔融旋甩、燃烧合成、放电等离子烧结等, 以及运 用各种纳米结构化手段和能谷调控手段协同实现热 电材料性能的提升 ${ }^{[5-8]}$ 。热电材料已经成为与电池、 光电材料、超级电容、光催化材料等具有相当地位 的新能源材料。

相比热电材料研究竞相追逐的局面, 热电器件 的相关研究却很少 ${ }^{[9-14]}$ 。近年来研发的大量新型热 电材料从实验室走向工程化, 实现大规模应用的案 例少之又少。本文将从 $\mathrm{Bi}_{2} \mathrm{Te}_{3}$ 型器件在温差发电方 面所遇到的技术瓶颈为例, 阐述热电器件中的界面 关键技术问题, 并对基于 $\mathrm{Bi}_{2} \mathrm{Te}_{3} 、 \mathrm{PbTe} 、 \mathrm{CoSb}_{3}$ 材料 的热电器件中界面封装和界面材料设计的研究进展 进行介绍, 并对未来热电器件所需热电界面材料提 出思考。

\section{1 热电器件的焊接工艺}

如图 1(a)所示, 热电器件通常包括 $\mathrm{p}$ 型、 $\mathrm{n}$ 型热 电半导体材料、金属电极、陶瓷基板以及外接金属 引线等几个部分, 其中陶瓷板提供器件的结构稳定 性和热电臂的阵列排布。金属电极通过串联的方式 把所有 $\mathrm{p}$ 型、 $\mathrm{n}$ 型热电臂交替串联连接形成电流通 路。图 1(b)是电极与热电材料间的电极接触界面的 放大示意图, 主要包含焊料层和金属化层。目前, 在 热电器件的封装过程中, 电极与热电材料间主要是通 过锡焊(Soldering)或钎焊(Brazing)的方式来连接 ${ }^{[15]}$ 。 表 1 是一些不同温区的常见焊料成分及其液固相温 度 ${ }^{[16]} 。 \mathrm{Bi}_{2} \mathrm{Te}_{3}$ 器件常用的焊料有 $\mathrm{Pb}_{37} \mathrm{Sn}_{63}, \mathrm{Bi}_{58} \mathrm{Sn}_{42}$ 和 $\mathrm{Sn}_{91.5} \mathrm{Sb}_{8.5}$ 。 由于大多数半导体材料与焊料间的润 湿性能不佳, 无法直接焊接, 因此焊接前需要在热 电臂两个端面先做金属化处理。 $\mathrm{Ni}$ 具有良好焊接性 能，最常被选用为金属化层材料。常见用于沉积 $\mathrm{Ni}$ 薄膜的方法包括: 磁控溅射、电化学沉积、无极电 镀等工艺。在热电半导体制冷工业中, 电化学沉积 (a)

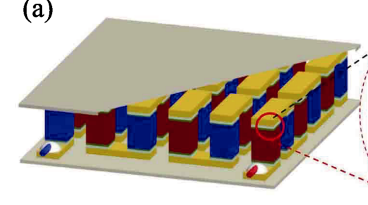

(b)

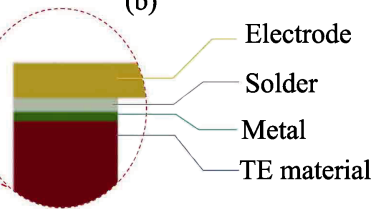

图 1 热电器件结构示意图(a)和电极界面结构示意图(b) Fig. 1 Schematic diagram of structure of thermoelectric device (a) and electrode interface (b)

表 1 一些焊料的成分和液相线、固相线温度 ${ }^{[16]}$

Table 1 Compositions of some solders as well as the temperature $(T)$ at liquidus and solidus ${ }^{[16]}$

\begin{tabular}{|c|c|c|c|}
\hline $\begin{array}{c}T \text { range } \\
/{ }^{\circ} \mathrm{C}\end{array}$ & Compositions/wt $\%$ & $\begin{array}{l}\text { Liquidus } \\
T /{ }^{\circ} \mathrm{C}\end{array}$ & $\begin{array}{c}\text { Solidus } \\
T /{ }^{\circ} \mathrm{C}\end{array}$ \\
\hline \multirow{4}{*}{$100-200$} & $52 \mathrm{In}+48 \mathrm{Sn}$ & 118 & 118 \\
\hline & $85 \mathrm{Sn}+10 \mathrm{Bi}+5 \mathrm{Zn}$ & 190 & 168 \\
\hline & $63 \mathrm{Sn}+37 \mathrm{~Pb}$ & 183 & 183 \\
\hline & $91.2 \mathrm{Sn}+8.8 \mathrm{Zn}$ & 198.5 & 198.5 \\
\hline \multirow{3}{*}{$200-300$} & $50 \mathrm{Sn}+50 \mathrm{~Pb}$ & 212 & 183 \\
\hline & $96.5 \mathrm{Sn}+3 \mathrm{Ag}+0.5 \mathrm{Cu}$ & 220 & 217 \\
\hline & $95 \mathrm{Sn}+5 \mathrm{Sb}$ & 240 & 232 \\
\hline \multirow{3}{*}{$300-400$} & $5 \mathrm{Sn}+95 \mathrm{~Pb}$ & 312 & 305 \\
\hline & $95 \mathrm{~Pb}+5 \mathrm{Ag}$ & 364 & 305 \\
\hline & $\begin{array}{l}75 \mathrm{Sn}+0.25 \mathrm{Sb}+ \\
0.25 \mathrm{Bi}+24.5 \mathrm{~Pb}\end{array}$ & 380 & 370 \\
\hline \multirow{2}{*}{$400-500$} & $94 \mathrm{Sn}+0.2 \mathrm{~Pb}+5.8 \mathrm{Sb}$ & 461 & 450 \\
\hline & $88 \mathrm{~Pb}+11.75 \mathrm{Sb}+0.25 \mathrm{Bi}$ & 473 & 473 \\
\hline \multirow{2}{*}{$500-600$} & $\begin{array}{c}97 \mathrm{~Pb}+0.4 \mathrm{Sb}+ \\
2.35 \mathrm{Ag}+0.25 \mathrm{Bi}\end{array}$ & 580 & 580 \\
\hline & $8.5 \mathrm{Sn}+90 \mathrm{~Pb}+1.5 \mathrm{Ag}$ & 588 & 588 \\
\hline
\end{tabular}


工艺是最常见的方法。金属化层的另外一个作用就 是阻止焊料元素扩散进入热电材料内部，以 $\mathrm{Bi}_{2} \mathrm{Te}_{3}$ 基器件为例, 焊料中的 $\mathrm{Sn}$ 或 $\mathrm{Pb}$ 扩散进入 $\mathrm{Bi}_{2} \mathrm{Te}_{3}$ 热 电臂将会显著改变材料的热电性能, 因此金属层需 要有一定厚度。根据不同的使用目的, $\mathrm{Ni}$ 层厚度约 在 $0.5 \sim 10 \mu \mathrm{m}$ 的范围。

\section{2 热电器件的界面问题}

近年来，随着航空航天以及新能源技术的需求, 热电器件在发电领域应用也逐渐受到人们关注。然 而，当人们尝试以传统热电制冷片进行发电应用时， 热电器件很快就失效了。 Hatzikraniotis 等 ${ }^{[17]}$ 将 Melcor HT9-3-25 型号 $\mathrm{Bi}_{2} \mathrm{Te}_{3}$ 器件用于温差发电, 冷 端固定在 $24{ }^{\circ} \mathrm{C}$, 热端经历 $30 \sim 200{ }^{\circ} \mathrm{C}$ 热循环测试, 发现输出功率持续缓慢下降, 6000 次循环 $(3000 \mathrm{~h})$ 后, 功率下降了 $14 \%$, 开路电压降低 $3.3 \%$, 内阻增 加 $16.1 \%$, 发现焊料和热电臂之间出现了微裂纹, 如图 2 所示。类似地, Park 等 ${ }^{[18]}$ 将 Ferrotec 9500/ 127/060 型号 $\mathrm{Bi}_{2} \mathrm{Te}_{3}$ 器件用于温差发电, 热端经历 $30 \sim 160{ }^{\circ} \mathrm{C}$ 热循环, 循环 6000 次后输出功率下降 $11 \%$, 并且接触界面出现裂纹。图 3 是上海硅酸盐研 究所的研究人员对 $\mathrm{CoSb}_{3}$ 基热电发电器件 $600{ }^{\circ} \mathrm{C}$ 弱 氧气氛老化 $12 \mathrm{~h}$ 后的失效分析结果, 从图中可以看 出, 失效的主要原因是金属化层与热电材料界面出现 开裂，同时 $\mathrm{p}$ 型 $\mathrm{CeFe}_{3.9} \mathrm{Mn}_{0.1} \mathrm{Sb}_{12}$ 铁元素氧化。金属化 层与热电材料界面是目前制约热电器件发展的瓶颈 技术，该界面也被称为电极接触界面或接触界面。

导致传统工艺封装的 $\mathrm{Bi}_{2} \mathrm{Te}_{3}$ 热电器件失效的主 要原因之一是 $\mathrm{Ni} / \mathrm{Bi}_{2} \mathrm{Te}_{3}$ 的界面结合强度不够。利用

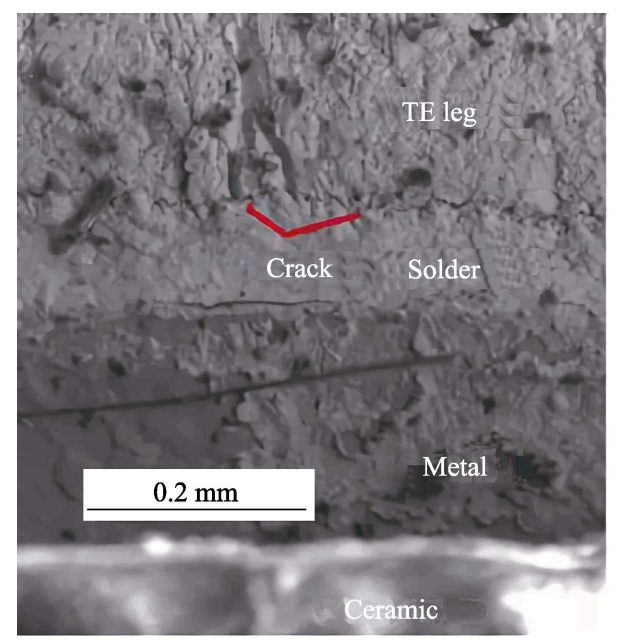

图 2 商业 $\mathrm{Bi}_{2} \mathrm{Te}_{3}$ 制冷片用于温差发电热循环测试后接触界 面的形貌 ${ }^{[17]}$

Fig. 2 Interface micrograph of commercial $\mathrm{Bi}_{2} \mathrm{Te}_{3}$ refrigeration device after the thermal cycle test ${ }^{[17]}$
磁控溅射、电化学沉积、无极电镀等工艺制备的 $\mathrm{Ni} / \mathrm{Bi}_{2} \mathrm{Te}_{3}$ 的界面结合强度约 8 10 MPa。对于热电发 电器件, 冷热端面的温差比传统制冷器件大很多, 界面热应力也相应地大很多 ${ }^{[19-20]}$ 。波士顿 GMZ 公 司的研究人员改进 $\mathrm{Ni} / \mathrm{Bi}_{2} \mathrm{Te}_{3}$ 界面连接工艺, 通过粉 末冶金的方式直接把 $\mathrm{Ni}$ 粉末烧结于端面，这样所获 得 $\mathrm{Ni} / \mathrm{Bi}_{2} \mathrm{Te}_{3}$ 的界面结合强度可以达到 20 30 MPa, 足以抵抗 $\mathrm{Bi}_{2} \mathrm{Te}_{3}$ 热端面在室温与 $200{ }^{\circ} \mathrm{C}$ 以上温度的 循环热震实验。但是通过扫描探针测试界面电阻, 发现 $\mathrm{Ni} / \mathrm{Bi}_{2} \mathrm{Te}_{2.7} \mathrm{Se}_{0.3}$ 的界面电阻率 $\left(210 \mu \Omega \cdot \mathrm{cm}^{2}\right)$ 比传 统电化学沉积方法制备样品 $\left(\sim 5 \mu \Omega \cdot \mathrm{cm}^{2}\right)$ 增加了 40 倍, 而 $\mathrm{Ni} / \mathrm{Bi}_{0.4} \mathrm{Sb}_{1.6} \mathrm{Te}_{3}$ 界面却保持接近理想的欧姆 接触 $\left(\sim 1 \mu \Omega \cdot \mathrm{cm}^{2}\right)$, 如图 4 所示 ${ }^{[21] 。}$

$\mathrm{Ni}$ 除了润湿焊料方便焊接外, 还有阻挡层的作 用，防止焊料中的 $\mathrm{Pb} 、 \mathrm{Sn}$ 等元素扩散进入 $\mathrm{Bi}_{2} \mathrm{Te}_{3}$ 基体。然而根据 $\mathrm{Lan}$ 等 ${ }^{[22]}$ 早期研究, $\mathrm{Ni}$ 在高温下也 会扩散进入 $\mathrm{Bi}_{2} \mathrm{Te}_{3}$ 。图 5 是 $\mathrm{Ni} / \mathrm{Bi}_{2} \mathrm{Te}_{2.7} \mathrm{Se}_{0.3}$ 和 $\mathrm{Ni} / \mathrm{Bi}_{0.4} \mathrm{Sb}_{1.6} \mathrm{Te}_{3}$ 界面附近成分分析 ${ }^{[21]}$ 。在两个界面 都发现形成了 $\mathrm{Ni}-\mathrm{Te}$ 化合物(如: $\mathrm{NiTe}$ 和 $\mathrm{Ni}_{2} \mathrm{Te}_{3}$ ), 界 面反应有利于获得高结合强度。通过合成验证，发 现 Ni-Te 化合物都是半金属, 室温电阻率 $<1 \mu \Omega \cdot \mathrm{m}$, 因此不是 $\mathrm{Ni} / \mathrm{Bi}_{2} \mathrm{Te}_{2.7} \mathrm{Se}_{0.3}$ 的界面电阻高的原因。进 一步的成分分析发现，在 $\mathrm{Ni} / \mathrm{Bi}_{2} \mathrm{Te}_{2.7} \mathrm{Se}_{0.3}$ 界面靠近 $\mathrm{Bi}_{2} \mathrm{Te}_{2.7} \mathrm{Se}_{0.3}$ 端, 出现一个 $\left(\mathrm{Bi}_{1-x} \mathrm{Ni}_{x}\right)_{2}\left(\mathrm{Te}_{2.7} \mathrm{Se}_{0.3}\right)_{3-\delta}$ 区 间。通过实验发现，当同时存在 $\mathrm{Ni}$ 掺杂和 $\mathrm{Te}$ 空位缺 陷时, $\left(\mathrm{Bi}_{1-x} \mathrm{Ni}_{x}\right)_{2}\left(\mathrm{Te}_{2.7} \mathrm{Se}_{0.3}\right)_{3-\delta}$ 会从通常的 $\mathrm{n}$ 型转变为 $\mathrm{p}$ 型并表现高电阻行为, 相关研究参考文献[21]。图 6 是相应的界面反应模型，界面反应在 $\mathrm{Ni} / \mathrm{Bi}_{2} \mathrm{Te}_{2.7} \mathrm{Se}_{0.3}$ 出现 Ni-Te 化合物以及 $\mathrm{p}$ 型 $\left(\mathrm{Bi}_{1-x} \mathrm{Ni}_{x}\right)_{2}\left(\mathrm{Te}_{2.7} \mathrm{Se}_{0.3}\right)_{3-\delta}$, 从而导致界面电阻急剧增加, 而 $\mathrm{Ni}$ 掺杂效应在 $\mathrm{p}$ 型 $\mathrm{Ni} / \mathrm{Bi}_{0.4} \mathrm{Sb}_{1.6} \mathrm{Te}_{3}$ 反而有利于降低接触电阻。并且由于 $\mathrm{p}$ 型 $\mathrm{Bi}_{0.4} \mathrm{Sb}_{1.6} \mathrm{Te}_{3}$ 材料中阳离子位上 $\mathrm{Sb}$ 占多数, 使得 $\mathrm{Te}$ 空位的数量也减少, 对 $\mathrm{Ni}$ 的扩散有针化作用。
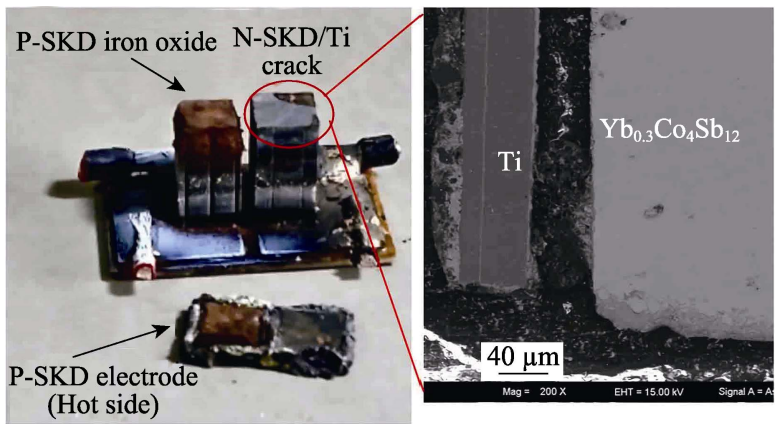

图 3 方钴矿器件热电偶臂的界面开裂与氧化(图片由上海 硅酸盐研究所热电材料器件课题组提供)(Skutterudite, SKD) Fig. 3 Interface cracking and oxidation images of Skutterudite thermoelectric legs 

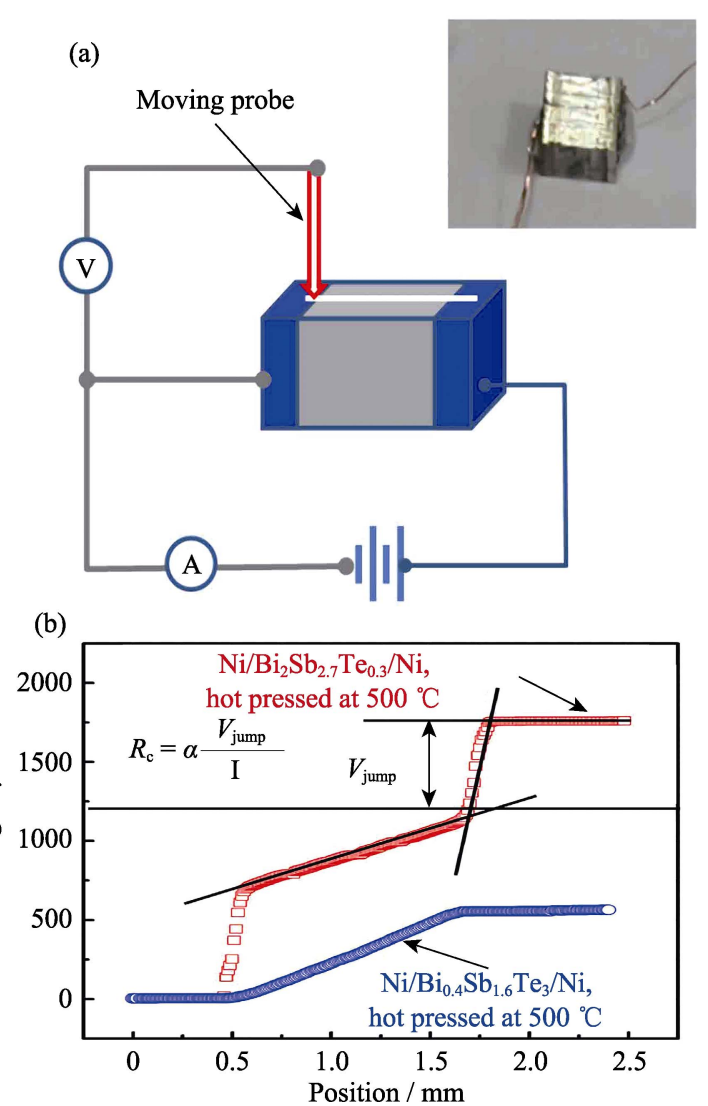

图 4 通过粉末热压法连接的 $\mathrm{Ni} / \mathrm{Bi}_{2} \mathrm{Te}_{3}$ 界面接触电阻的扫 描探针测试方法示意图(a)(插图为 $\mathrm{Bi}_{2} \mathrm{Te}_{3}$ 基接触脚照片), $\mathrm{Ni} / \mathrm{Bi}_{2} \mathrm{Te}_{2.7} \mathrm{Se}_{0.3} / \mathrm{Ni}$ 和 $\mathrm{Ni} / \mathrm{Bi}_{0.4} \mathrm{Sb}_{1.6} \mathrm{Te}_{3} / \mathrm{Ni}$ 的电势曲线以及接触 电阻计算方法(b) ${ }^{[21]}$

Fig. 4 Schematic diagram of a scanning voltage probe for contact resistance measurement, and $\mathrm{a}_{2} \mathrm{Te}_{3}$-based leg (inset) (a); contact resistance measurement for both n-type $\mathrm{Ni} / \mathrm{Bi}_{2} \mathrm{Te}_{2.7} \mathrm{Se}_{0.3} / \mathrm{Ni}$ and p-type $\mathrm{Ni} / \mathrm{Bi}_{0.4} \mathrm{Sb}_{1.6} \mathrm{Te}_{3} / \mathrm{Ni}(\mathrm{b})^{[21]}$

因此, 解决 $\mathrm{Ni} / \mathrm{Bi}_{2} \mathrm{Te}_{2.7} \mathrm{Se}_{0.3}$ 界面问题的关键是 避免出现 $\mathrm{p}$ 型 $\left(\mathrm{Bi}_{1-x} \mathrm{Ni}_{x}\right)_{2}\left(\mathrm{Te}_{2.7} \mathrm{Se}_{0.3}\right)_{3-\delta}$ 区。可在 $\mathrm{Ni}$ 与 $\mathrm{Bi}_{2} \mathrm{Te}_{2.7} \mathrm{Se}_{0.3}$ 间加入钝化 $\mathrm{Ni}$ 扩散的 $\mathrm{n}$ 型重掺杂层。 如果金属 $\mathrm{Ni}$ 与 $\mathrm{Bi}_{2} \mathrm{Te}_{2.7} \mathrm{Se}_{0.3}$ 间完全没有扩散, 则结 合强度无法满足要求。比如, 通过加入 $\mathrm{n}$ 型重掺杂 层 $\left(\mathrm{SbI}_{3}\right.$ 掺杂的 $\left.\mathrm{Bi}_{2} \mathrm{Te}_{2.7} \mathrm{Se}_{0.3}\left(\mathrm{n}^{+}-\mathrm{BTS}\right)\right)$ 所制备的 $\mathrm{Ni} / \mathrm{n}^{+}-\mathrm{BTS} / \mathrm{n}-\mathrm{BTS}$, 界面电阻率 $<1 \mu \Omega \cdot \mathrm{cm}^{2}$, 结合强 度 $16 \mathrm{MPa}$; 运行 $150 \mathrm{~h}$, 器件电阻变化 $<1 \%$ 。但通 过粉末冶金工艺连接时, $\mathrm{n}^{+}$-BTS 层厚度很难做到小 于 $100 \mu \mathrm{m}$ 。由于 $\mathrm{n}^{+}-\mathrm{BTS}$ 的热电性能远比正常的 $\mathrm{n}-\mathrm{BTS}$ 差, 所以 $\mathrm{n}^{+}-\mathrm{BTS}$ 过渡层对器件的效率仍有较 大负面影响。因此, Liu 等 ${ }^{[21]}$ 又进一步开发了 $\mathrm{NiFe}$ 基合金, 作为 $\mathrm{n}$ 型 $\mathrm{Bi}_{2} \mathrm{Te}_{2.7} \mathrm{Se}_{0.3}$ 的金属化层材料, 该 界面在室温到 $250{ }^{\circ} \mathrm{C}$ 温度区间循环热震测试 12 次 后, 接触电阻率 $\left(<5 \mu \Omega \cdot \mathrm{cm}^{2}\right)$ 仍保持不变。该电极界 面工艺在第二代太阳能热电发电器件中得到了应用, 如图 7 示 $^{[12]}$ 。

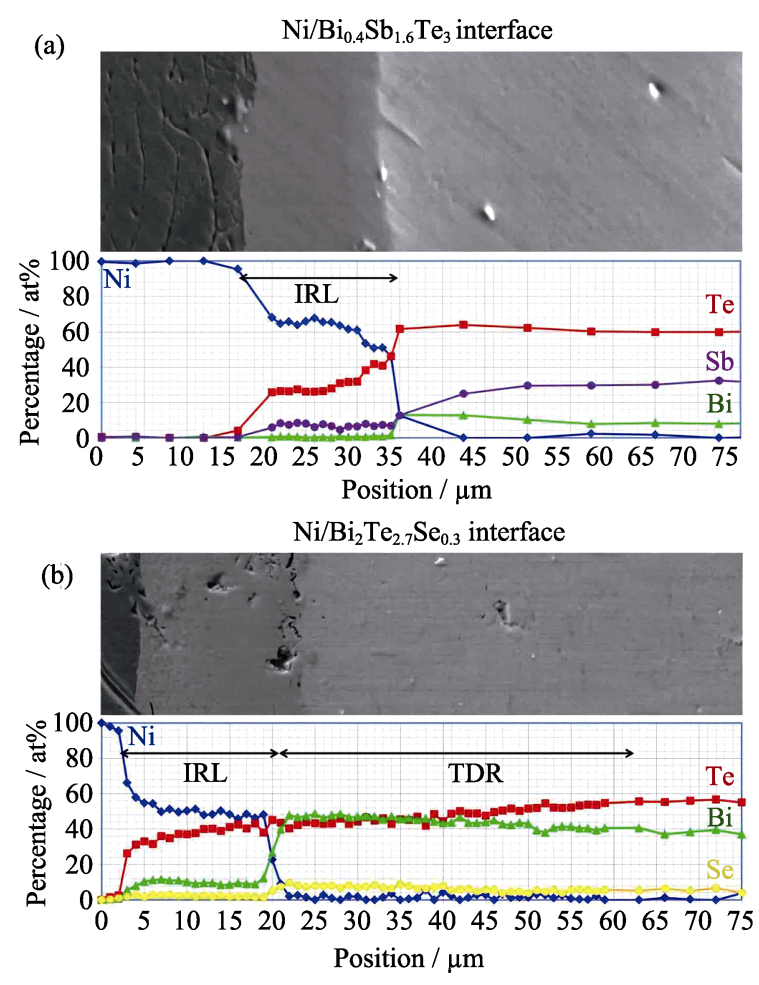

图 $5 \mathrm{Ni} / \mathrm{Bi}_{0.4} \mathrm{Sb}_{1.6} \mathrm{Te}_{3}$ 界面(a)和 $\mathrm{Ni} / \mathrm{Bi}_{2} \mathrm{Te}_{2.7} \mathrm{Se}_{0.3}$ 界面(b)附近 元素百分浓度分布 ${ }^{[21]}$

Fig. 5 Comparison of composition profile between $\mathrm{Ni} / \mathrm{Bi}_{0.4} \mathrm{Sb}_{1.6} \mathrm{Te}_{3}$ interface (a) and $\mathrm{Ni} / \mathrm{Bi}_{2} \mathrm{Te}_{2.7} \mathrm{Se}_{0.3}$ Interface (b) obtained from a selected area SEM-EDS ${ }^{[21]}$

IRL: interface reaction layer, TDR: Te-deficient region

\begin{tabular}{|c|c|c|}
\hline \multicolumn{3}{|c|}{ Before hot press } \\
\hline $\mathrm{Ni}$ & & $(\mathrm{Bi}, \mathrm{Sb})_{2}(\mathrm{Te}, \mathrm{Se})_{3}$ \\
\hline \multicolumn{3}{|c|}{ After hot press } \\
\hline $\mathrm{Ni}$ & $\mathrm{Ni}_{3} \mathrm{Te}_{2}, \mathrm{NiTe}$ & $\left(\mathrm{Bi}_{1-x} \mathrm{Ni}_{\mathrm{x}}\right)_{2}(\mathrm{Te}, \mathrm{Se})_{3-\delta} \quad \mathrm{Bi}_{2}(\mathrm{Te}, \mathrm{Se})_{3}$ \\
\hline \multicolumn{3}{|c|}{$\mathrm{p}$-type leg: defect $\mathrm{Ni}_{\mathrm{T}}$, increase the $\mathrm{p}$-type carrier $\rightarrow \mathrm{no} R_{\mathrm{c}}$} \\
\hline $\mathrm{Ni}$ & $\mathrm{Ni}_{3} \mathrm{Te}_{2}, \mathrm{NiTe}$ & $(\mathrm{Bi}, \mathrm{Sb})_{2} \mathrm{Te}_{3}$ \\
\hline
\end{tabular}

$\left(\mathrm{Sb}, \mathrm{Bi}, \mathrm{Ni}_{x}\right)_{2} \mathrm{Te}_{3}$

图 $6 \mathrm{Bi}_{2} \mathrm{Te}_{3}$ 基热电臂电极界面高温反应模型 ${ }^{[21]}$

Fig. 6 High-temperature reaction model of electrode interface of $\mathrm{Bi}_{2} \mathrm{Te}_{3}$-based thermoelectric leg ${ }^{[21]}$

\section{3 界面对器件性能的影响}

前述通过实例阐述了金属化界面是热电器件关 键界面, 结合强度决定了热电发电器件是否机械可 靠。同时也注意到, 由于连接工艺和金属层材料(或 称为热电界面材料)的差异, 界面电阻可能会有数 量级的差异。下文将理论分析界面电阻和界面热阻 对热电发电器件功率和效率的影响。

考虑器件的界面热阻和界面电阻, 同时假定 $\mathrm{p}$ 

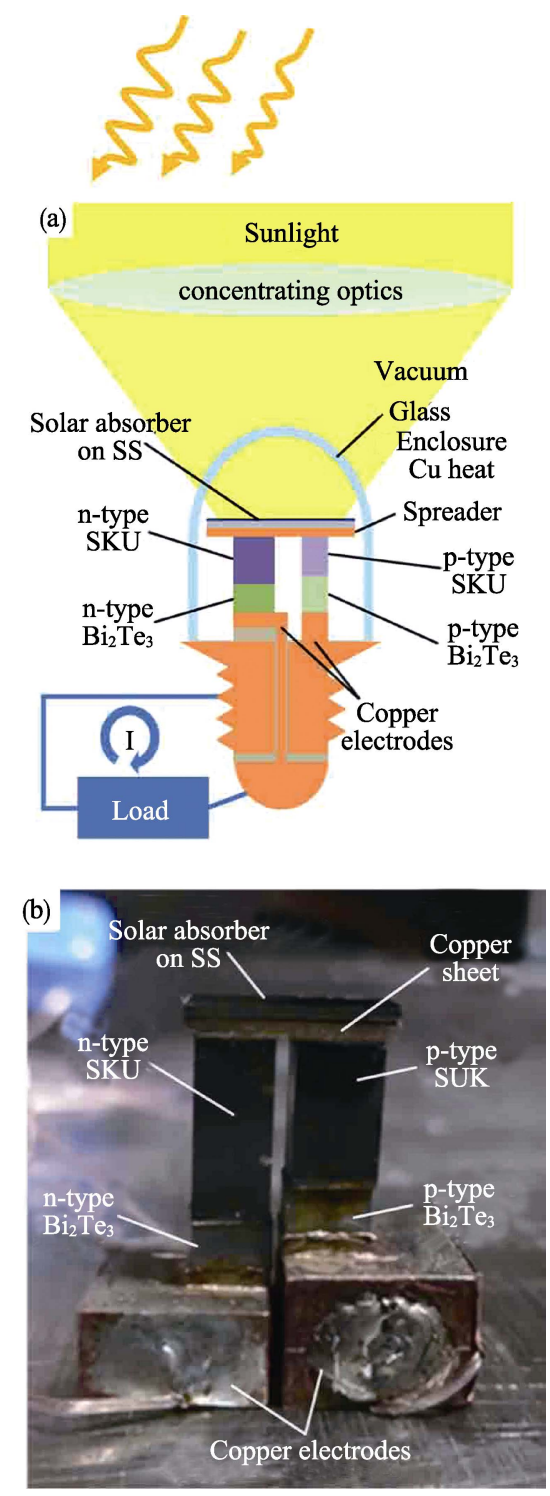

图 7 聚光太阳能热电发电器件: 新型 $\mathrm{NiFe}$ 基合金用于 $\mathrm{n}$ 型 $\mathrm{Bi}_{2} \mathrm{Te}_{3}$ 的金属化 ${ }^{[12]}$

Fig. 7 Concentrating solar thermoelectric generators: new type of NiFe-based alloy applied to metallization of n-type $\mathrm{Bi}_{2} \mathrm{Te}_{3}{ }^{[12]}$

型和 $\mathrm{n}$ 型材料有着相同的热导率和电阻率数值, 那 么热电发电器件的开路电压 $U_{\max }$ 和最优工作电流 $I$ 可以简单通过式(2)和式(3)描述, 其中 $T_{\mathrm{h}} 、 T_{\mathrm{c}}$ 为上下 电极温度, $S_{\mathrm{p}} 、 S_{\mathrm{n}}$ 为 $\mathrm{p} 、 \mathrm{n}$ 热电臂的塞贝克系数, $N$ 为 $\mathrm{p} 、 \mathrm{n}$ 热电偶臂数目, $R_{\mathrm{Th}} 、 R_{\mathrm{iTh}}$ 为热电臂的热阻与 接触热阻, $R_{\mathrm{EI}} 、 R_{\mathrm{iEl}}$ 分别为热电臂的电阻与接触电 阻。从式(2)可知, 界面热阻会降低开路电压, 因为 热电材料两端的实际温差稍小于上下电极温差; 如 果再考虑陶瓷基板和电极间的热阻，那么作用于热 电材料两端的实际温差明显低于基板两侧的温差。 当内外电阻相同时, 器件具有最高输出功率, 获得 最优工作电流。从式(3)可知, 界面电阻会降低输出 电流。虽然材料的电导率可以在很大的数量级区间
内变动, 但是热电半导体的电导率一般处于 $10^{4} \sim 10^{5} \mathrm{~S} \cdot \mathrm{m}^{-1}$ 量级, 因此界面材料的电导率也必须 $\geqslant 10^{4} \sim 10^{5} \mathrm{~S} \cdot \mathrm{m}^{-1}$ 量级。如果界面厚度为 $10 \sim 100 \mu \mathrm{m}$, 那么界面的接触电阻率要求 $\leqslant 10 \mu \Omega \cdot \mathrm{cm}^{2}$ 。

$$
\begin{gathered}
U_{\max }=\frac{N\left(S_{\mathrm{p}}-S_{\mathrm{n}}\right)\left(T_{\mathrm{h}}-T_{\mathrm{c}}\right)}{1+2 \frac{R_{\mathrm{iTh}}}{R_{\mathrm{Th}}}} \\
I=\frac{N\left(S_{\mathrm{p}}-S_{\mathrm{n}}\right)\left(\mathrm{T}_{\mathrm{h}}-\mathrm{T}_{\mathrm{c}}\right)}{\left(1+2 \frac{R_{\mathrm{iTh}}}{R_{\mathrm{Th}}}\right) \cdot\left[2 N\left(4 R_{\mathrm{iEl}}+2 \mathrm{R}_{\mathrm{El}}\right)\right]}
\end{gathered}
$$

相比于界面热阻, 界面电阻更能显著影响器件 性能。器件性能恶化往往是由界面电阻增加引起的, 进而导致输出电流降低; 界面电阻的大幅增加会造 成器件彻底失效。为了更好地揭示出界面接触电阻 对器件的发电功率和效率的影响, 忽略所有寄生热 阻, 假定器件由碲化铋单一热电偶臂组成, 并且材 料的三个热电参数为常数值, 那么当负载和内阻匹 配时获得的最大输出功率和效率可用式(4)和式(5) 表示 ${ }^{[23]}$, 其中 $A$ 为热电臂横截面积, $l$ 为热电材料高 度, $\rho$ 为热电材料的电阻率, $\rho_{\mathrm{iEl}}$ 为界面处的接触电 阻率 $\left(\rho_{\mathrm{iEl}}=R_{\mathrm{iEl}} \cdot A, \mu \Omega \cdot \mathrm{cm}^{2}\right), \eta_{\mathrm{c}}$ 为卡诺效率。

$$
\begin{gathered}
P_{\max }=\frac{\left(S_{\mathrm{p}}-S_{\mathrm{n}}\right)^{2}\left(T_{\mathrm{h}}-T_{\mathrm{c}}\right)^{2}}{4\left(\frac{4 \rho_{\mathrm{iEl}}}{A}+\frac{2 \rho l}{A}\right)} \\
\eta_{P_{\text {max }}}=\eta_{\mathrm{c}} /\left(2-0.5 \eta_{\mathrm{c}}+\frac{4}{Z_{\mathrm{M}} T_{\mathrm{h}}}\left(1+\frac{2 \rho_{\mathrm{iEI}}}{\rho l}\right)\right)
\end{gathered}
$$

进一步假定: $T_{\mathrm{h}}=503 \mathrm{~K}, T_{\mathrm{c}}=303 \mathrm{~K}, S_{\mathrm{p}}=$ $180 \mu \mathrm{V} \cdot \mathrm{K}^{-1}, S_{\mathrm{n}}=-180 \mu \mathrm{V} \cdot \mathrm{K}^{-1}, \rho=10 \mu \Omega \cdot \mathrm{m}, k=$ $1.2 \mathrm{~W} \cdot \mathrm{K}^{-1} \cdot \mathrm{m}^{-1}$, 热电臂尺寸为 $2 \mathrm{~mm} \times 2 \mathrm{~mm} \times l$ (高度)。 可以计算出不同热电臂高度 $l(l=1 \sim 5 \mathrm{~mm})$ 时, 接触 电阻率 $\rho_{\mathrm{iEl}}\left(0 \sim 200 \mu \Omega \cdot \mathrm{cm}^{2}\right)$ 如何降低最大输出功率 和效率, 如图 8 所示。图 8(a)可以看出, 随着接触电 阻率增大, 最大输出功率下降, 而且热电臂越短, 输出功率下降得越明显, 这是由于接触电阻占内阻 比例更大; 同时热电臂越短, 输出功率越大, 这是 由于总内阻变小而塞贝克电压保持不变。图 8(b)可 以看出, 接触电阻率为 0 时, 热电效率不随热电臂 长度不同而变化, 此时的效率由热电材料和卡诺效 率共同决定; 随着接触电阻率增大, 效率均下降, 这是因为输出功率下降。另外, 相同接触电阻率条 件下, 热电臂越短效率越低。在常见的热发电器件 中, 通常要求接触电阻率小于 $10 \mu \Omega \cdot \mathrm{cm}^{2}$, 以保证 器件的输出功率和效率不会明显降低。Zhang 等 ${ }^{[13]}$ 对 $\mathrm{Bi}_{2} \mathrm{Te}_{3} / \mathrm{SKD}$ 真实热电偶臂有限元计算结果支持 以上的理论分析: 总接触电阻率为 $40 \mu \Omega \cdot \mathrm{cm}^{2}$ 造成 

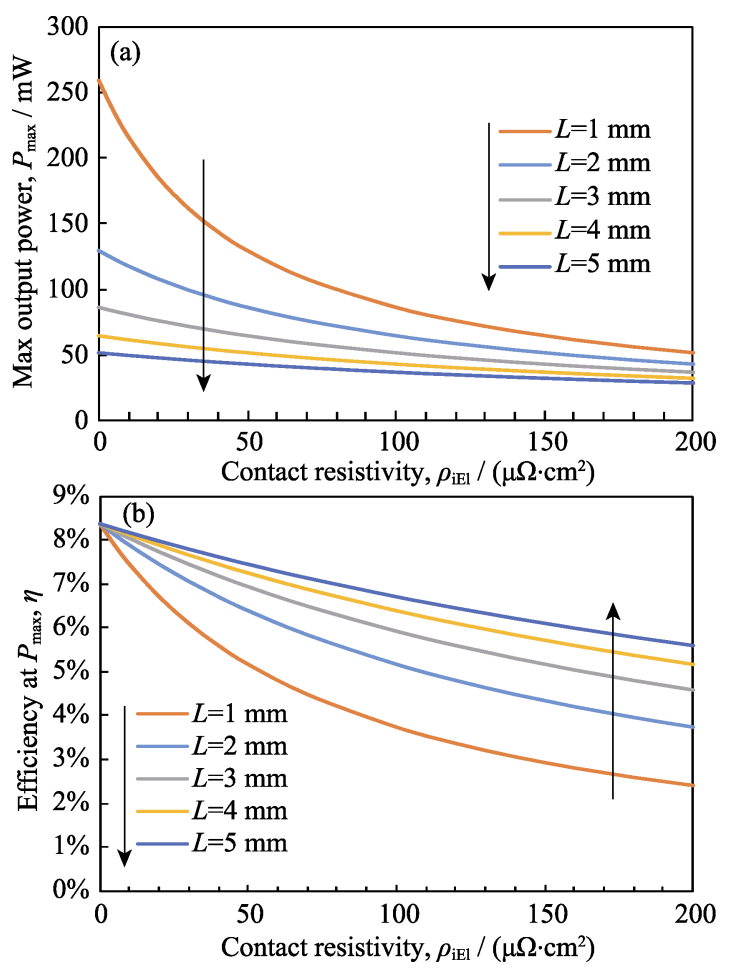

图 8 虚拟的碲化铋热电偶臂最大输出功率 (a)和效率(b)随 界面接触电阻率的变化情况, 其中 $l$ 为热电臂高度

Fig. 8 The maximum output power (a) and efficiency (b) $v s$. interface contact resistivity for the simulated $\mathrm{Bi}_{2} \mathrm{Te}_{3} \operatorname{leg}(l$ is the leg height)

的器件效率损失低于 $2 \%$, 其热电臂尺寸约为 $4 \mathrm{~mm} \times$ $4 \mathrm{~mm} \times 12 \mathrm{~mm}$, 包含 6 个接触界面。

热电材料和金属的接触电阻率取决于界面电子 结构, 这可以采用经典的金属-半导体接触能带理 论进行分析 ${ }^{[24]}$ 。接触电阻率与功函数紧密相关。对 于 $\mathrm{n}$ 型材料, 当金属的功函数大于热电材料功函数 时, 界面处的肖特基势垒造成接触电阻, 功函数差 别越大，肖特基势垒就越高，接触电阻也就越大; 当金属的功函数小于热电材料功函数时, 界面形成 欧姆接触，接触电阻很小。对于 $\mathrm{p}$ 型材料，当金属的 功函数小于热电材料功函数时, 界面处肖特基势垒 造成接触电阻; 当金属的功函数大于热电材料功函 数时, 界面形成欧姆接触, 接触电阻很小。因此, 热 电材料的金属化也需要考虑热电材料和金属功函数 的匹配。

\section{4 热电器件的界面研究现状}

\section{1 碲化铋基热电材料的界面}

应用于热电制冷器件, $\mathrm{Bi}_{2} \mathrm{Te}_{3}$ 基热电材料的界 面制造工艺是逐渐成熟的。人们早期用锡焊将 $\mathrm{Bi}_{2} \mathrm{Te}_{3}$ 基材料与铜金属直接连接。例如, $\mathrm{Haba}$ 等 ${ }^{[25]}$ 通过增加表面粗楉度并进行无机盐甲醇溶液处理改
善了 $\mathrm{p}$ 型、 $\mathrm{n}$ 型材料与 $\mathrm{Cu}$ 的焊锡润湿性能，随后采 用 $\mathrm{Sn}_{0.475} \mathrm{Bi}_{0.5} \mathrm{Sb}_{0.025}\left(266 \sim 274{ }^{\circ} \mathrm{C}\right)$ 和 $\mathrm{Sn}_{0.6} \mathrm{~Pb}_{0.4}$ 分别涂 覆 $\mathrm{Bi}_{2} \mathrm{Te}_{3}$ 和铜表面, 实现两种材料界面连接 $\left(230{ }^{\circ} \mathrm{C}\right)$ 。 然而, $\mathrm{Sn}$ 在 $\mathrm{n}$ 型 $\mathrm{Bi}_{2} \mathrm{Te}_{3}$ 内的扩散增加了内阻, 导致 器件效率降低 $10 \%$ 。 Rosi 与 Bernoff 等 ${ }^{[26]}$ 采用 $\mathrm{Sb}_{0.48} \mathrm{Ag}_{0.52}\left(490 \sim 520{ }^{\circ} \mathrm{C}\right)$ 和 $\mathrm{Sb}_{0.45} \mathrm{Ag}_{0.55}\left(500{ }^{\circ} \mathrm{C}\right)$ 无锡 焊接 $\mathrm{Bi}_{2} \mathrm{Te}_{3}$ 和 $\mathrm{Cu}$, 获得了 $200 \mu \Omega$ 界面电阻, 但未能给 出界面电阻率，同时 $\mathrm{Ag}$ 的扩散也造成 $\mathrm{n}$ 型 材料热电 性能恶化。Liao 等 ${ }^{[27]}$ 使用 $\mathrm{Sn}_{0.63} \mathrm{~Pb}_{0.37} 、 \mathrm{Sn}_{0.955} \mathrm{Ag}_{0.04} \mathrm{Au}_{0.005}$ 焊料, 分别实现 $\mathrm{p}$ 型、 $\mathrm{n}$ 型 $\mathrm{Bi}_{2} \mathrm{Te}_{3}$ 与铜电极互联, 他 们发现界面处生成了 $\mathrm{SnTe}$ 与 $\mathrm{Pb}_{1-x} \mathrm{Sn}_{x} \mathrm{Te}$ 化合物, 其界 面电阻率 $10 \sim 100 \mu \Omega \cdot \mathrm{cm}^{2}$, 与化合物组成、厚度相关。

为了避免焊锡的负面影响, 人们采用 $\mathrm{Bi}_{2} \mathrm{Te}_{3}$ 基 热电材料的 $\mathrm{Ni}$ 金属化工艺。 $\mathrm{Ni}$ 金属化工艺分成化 学法和物理法两种, 包括化学镀、电化学沉积和溅 射、喷涂等。另外，热压工艺也可用于 $\mathrm{Ni}$ 金属化，提 高 $\mathrm{Ni}$ 金属层的结合强度和厚度。金属化 $\mathrm{Ni}$ 的薄膜 质量和基材表面状况、制备工艺参数密切有关。Lan 等 ${ }^{[22]}$ 发现 $\mathrm{Ni}$ 作为阻挡层可以抑制 $\mathrm{Sn}$ 往 $\mathrm{Bi}_{2} \mathrm{Te}_{3}$ 材料 的扩散, 如果无金属化, 元素 $\mathrm{Sn}$ 可扩散至 $\mathrm{Bi}_{2} \mathrm{Te}_{3}$ 材质 $4 \mu \mathrm{m}$ 深度 $\left(200 \sim 230{ }^{\circ} \mathrm{C}, 1 \sim 2 \mathrm{~min}\right)$, 并且相同条 件下 $\mathrm{Ni}$ 在 $\mathrm{n}$ 型 $\mathrm{Bi}_{2} \mathrm{Te}_{3}$ 比在 $\mathrm{p}$ 型扩散更深入, 分别为 $3 \mu \mathrm{m}$ 和 $100 \mathrm{~nm}$ 。对于电镀 $\mathrm{Ni}, \mathrm{Ni} / \mathrm{p}-\mathrm{Bi}_{2} \mathrm{Te}_{3}$ 接触电阻 率低至 $1.7 \sim 2.6 \mu \Omega \cdot \mathrm{cm}^{2}$, 而 $\mathrm{Ni} / \mathrm{n}-\mathrm{Bi}_{2} \mathrm{Te}_{3}$ 接触电阻率 为 7 10 $\mu \Omega \cdot \mathrm{cm}^{2[28]}$ 。为了增强 $\mathrm{Ni}$ 的粘附, Weitzman 等 ${ }^{[29]}$ 通过混酸刻蚀工艺增加 $\mathrm{Bi}_{2} \mathrm{Te}_{3}$ 表面粗楉度, 提 高了 $\mathrm{Ni} / \mathrm{p}-\mathrm{Bi}_{2} \mathrm{Te}_{3}$ 和 $\mathrm{Ni} / \mathrm{n}-\mathrm{Bi}_{2} \mathrm{Te}_{3}$ 界面结合强度, 分别 达到 7.2 10.6 MPa 和 9.7 12.2 MPa, 而传统喷砂表 面处理后的 $\mathrm{Ni}$ 层结合强度分别只有 $1.9 \sim 3.1 \mathrm{MPa}$ 和 3.1 6.7 $\mathrm{MPa}$ 。等离子清洁处理可以去除 $\mathrm{Bi}_{2} \mathrm{Te}_{3}$ 表层氧化物, 将溅射 $\mathrm{Ni}$ 接触电阻从 $100 \mu \Omega \cdot \mathrm{cm}^{2}$ 降

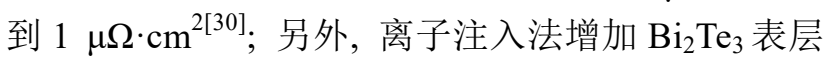
掺杂浓度可以获得更低的 $\mathrm{Ni} / \mathrm{Bi}_{2} \mathrm{Te}_{3}$ 接触电阻率 ${ }^{[30]}$ 。 Lin 等 ${ }^{[31]}$ 采用一种化学清洗工艺, 溶液为氨水和过 氧化氢水溶液, 清洗工艺为 $70{ }^{\circ} \mathrm{C} 、 15 \mathrm{~min}$, 最后采 用 $2 \% \mathrm{HF}$ 溶液去除表层氧化物。Iyore 等 ${ }^{[32]}$ 采用了 一种更加考究的清洗策略以便溅射沉积和化学镀 镍: 1)用丙酮蒸气、异丙醇、去离子水分别清洗, 氮 气吹干; 2)采用 $\mathrm{H}_{2} \mathrm{O}-\mathrm{H}_{2} \mathrm{O}_{2}-\mathrm{HCl}(2: 1: 1)$ 溶液清洗 $30 \mathrm{~s} ; 3$ )采用 $30 \%$ 氨水溶液清洗 $1 \mathrm{~min}$ 。步骤 2 )和 3) 能将接触电阻率从 $8 \mu \Omega \cdot \mathrm{cm}^{2}$ 降至 $3 \mu \Omega \cdot \mathrm{cm}^{2}$; 采用 粗粘度为 $3 \mathrm{~nm}$ ( $\mathrm{rms}$, 标准差)的 $\mathrm{Bi}_{2} \mathrm{Te}_{3}$ 抛光表面金 属化, 接触电阻率小于 $1 \mu \Omega \cdot \mathrm{cm}^{2}$, 但界面强度较 低。Feng 等 ${ }^{[33}$ 采用电解抛光和化学机械抛光 $\mathrm{Bi}_{2} \mathrm{Te}_{3}$, 获得 $1.9 \mathrm{~nm}(\mathrm{rms})$ 的表面粗糙度, 外加溴乙醇溶液和 特殊清洗剂去除氧化物和吸附颗粒。金属化后, 
$\mathrm{Ni} / \mathrm{p}-\mathrm{Bi}_{2} \mathrm{Te}_{3}$ 和 $\mathrm{Ni} / \mathrm{n}-\mathrm{Bi}_{2} \mathrm{Te}_{3}$ 的接触电阻率分别为 $0.3 \sim 1.1$ 和 $0.7 \sim 1.2 \mu \Omega \cdot \mathrm{cm}^{2}$ 。综合考虑接触电阻、结 合强度以及工艺简便性, 工业上 $\mathrm{Bi}_{2} \mathrm{Te}_{3}$ 材料 $\mathrm{Ni}$ 金属 化的一般工艺如下: 1)喷砂或刻蚀处理达到 $\mathrm{Bi}_{2} \mathrm{Te}_{3}$ 表面粗糙化; 2)化学清洗; 3) 电镀或化学镀 $\mathrm{Ni}$, 厚度 $0.5 \sim 10 \mu \mathrm{m}$ 。 Iyore 等 ${ }^{[22,34]}$ 研究了 $\mathrm{n}$ 型材料溅射 $\mathrm{Ni}$ 的界 面热稳定, 得到了 $\mathrm{Ni}$ 在 $\mathrm{Bi}_{2} \mathrm{Te}_{3}$ 内扩散系数, 即 $D\left(100{ }^{\circ} \mathrm{C}\right)=5 \times 10^{-20} \mathrm{~m}^{2} \cdot \mathrm{s}^{-1}, D\left(150{ }^{\circ} \mathrm{C}\right)=7 \times 10^{-19} \mathrm{~m}^{2} \cdot \mathrm{s}^{-1}$ 和 $D\left(200{ }^{\circ} \mathrm{C}\right)=6 \times 10^{-18} \mathrm{~m}^{2} \cdot \mathrm{s}^{-1}$, 给出扩散的活化温度 为 $100{ }^{\circ} \mathrm{C}$ 因此用于制冷应用时 $\mathrm{Ni} / \mathrm{Bi}_{2} \mathrm{Te}_{3}$ 界面稳定。

传统的商业 $\mathrm{Bi}_{2} \mathrm{Te}_{3}$ 器件如应用于温差发电, 除 了需要新的金属化层合金作为界面材料以外，还需 要考虑合适的焊料。为了输出尽可能大的发电功率, 器件热端处在 $200 \sim 250{ }^{\circ} \mathrm{C}$ 范围, 其相应焊料可以选 用 $\mathrm{Sn}-\mathrm{Sb}$ 焊料。

\section{$4.2 \mathrm{PbTe}$ 基热电材料的界面}

$\mathrm{PbTe}$ 热电材料用于温差发电技术可以追溯到 上世纪 60 年代, 为宇宙深空探测器供给持续、稳定、 可靠的电能, 它以同位素衰减热为热源, 实现了 $5 \%$ 6\%热电转换效率 ${ }^{[15]}$ 。铁用作 $\mathrm{PbTe}$ 的电极材料, 与 $\mathrm{n}$ 型 $\mathrm{PbTe}$ 接触电阻率小于 $10 \mu \Omega \cdot \mathrm{cm}^{2}$, 结合强度 甚至大于 $\mathrm{PbTe}$ 材料本身; 而 $\mathrm{p}$ 型 $\mathrm{PbTe}$ 采用 $\mathrm{SnTe}$ 半金属作为中间层与 $\mathrm{Fe}$ 连接后, 其接触电阻率也小 于 $10 \mu \Omega \cdot \mathrm{cm}^{2}{ }^{[35]}$ 。Singh 等 ${ }^{[36]}$ 同样采用 $\mathrm{Fe}$ 作为接触 材料, 添加 $200 \mu \mathrm{m}$ 厚 $0.5 \mathrm{PbTe}-0.5 \mathrm{Fe}$ 混合物作为缓 冲层 $600{ }^{\circ} \mathrm{C}$ 真空热压制作 $\mathrm{n}-\mathrm{PbTe}$ 热电臂, 添加 $\mathrm{SnTe}$ 中间层形成 p-TAGS-85 热电臂, 通过测量热电偶臂 的内阻推导出平均的界面电阻率小于 $7.6 \mu \Omega \cdot \mathrm{cm}^{2}$, 界面电阻占 $3.5 \%$ 的总内阻, 并且界面元素分布清晰 未发生扩散生成化合物。Leavitt 等 ${ }^{\left[{ }^{[7]}\right.}$ 申请的专利中 考虑了 $\mathrm{Fe}$ 和 $\mathrm{PbTe}$ 的热膨胀系数差异 $\left(12 \times 10^{-6}\right.$ 和 $18 \times 10^{-6} \mathrm{~K}^{-1}$ ), 采用 $1 \mathrm{~mm}$ 厚的 $0.75 \mathrm{PbTe}-0.25 \mathrm{Fe}$ 混合 粉末层作为 $\mathrm{p}$ 型、 $\mathrm{n}$ 型 $\mathrm{PbTe}$ 和 $\mathrm{Fe}$ 之间的缓冲, 然后 采用真空热压粉末治金工艺。Xia 等 ${ }^{[38-40]}$ 尝试了 $\mathrm{Fe} 、 \mathrm{Mo} 、 \mathrm{Ni} 、 \mathrm{NiFeMo} 、 \mathrm{Nb}$ 䈃与 $\mathrm{n}$ 型 $\mathrm{PbTe}$ 粉末热 压连接, Mo 未能成功键合; 扫描电镜观察了 $\mathrm{PbTe} / \mathrm{Fe}$ 界面, 发现 $\mathrm{Fe}$ 在 $\mathrm{PbTe}$ 内能有 $20 \mu \mathrm{m}$ 扩散深 度, 浓度依指数关系递减, 但未反应形成铁碲化合 物, 两相界面清晰, 无过渡层; 而 $\mathrm{Ni} 、 \mathrm{Nb}$ 连接生成 了 $\mathrm{Ni}_{3} \mathrm{Te}_{2}$ 和 $\mathrm{Nb}_{3} \mathrm{Te}_{4}$, 但研究未给出这些界面的接触 电阻数据。

此外, $\mathrm{Ni}$ 也被尝试用作 $\mathrm{PbTe}$ 的接触材料。 Orihashi 等 $^{[41]}$ 发现 $\mathrm{Ni} / \mathrm{n}$-PbTe 无明显接触电阻, 而 $\mathrm{Ni} / \mathrm{p}-\mathrm{Pb}_{0.5} \mathrm{Te}_{0.5}$ 界面可能由于生成了 $\mathrm{Ni}$ 施主能级有 着较大的接触电阻, 通过增加 $\mathrm{SnTe}$ 中间层可以降低 该电阻。放电等离子技术也用于一步烧结键合
$\mathrm{n}-\mathrm{PbTe}$ 粉末和 $\mathrm{Ni}$ 片，形成 $27 \mu \mathrm{m}$ 厚的 $\mathrm{Ni}_{3} \mathrm{Te}_{2}$ 中间层, 由 $\mathrm{Te}$ 扩散进入 $\mathrm{Ni}$ 反应生成, $\mathrm{Pb}$ 元素基本无迁移 ${ }^{[41-42]}$ 。 $\mathrm{Hu}$ 等 ${ }^{[11]}$ 采用 $\mathrm{Co}_{0.8} \mathrm{Fe}_{0.2}$ 粉末共烧结工艺代替纯铁制 作 $\mathrm{p}$ 型、 $\mathrm{n}$ 型 $\mathrm{PbTe}$ 的界面材料, 用其组装的原型器 件发电功率稳定, 说明界面电阻率稳定; 采用液态 金属软连接热电臂和铜电极, 比较热端 $600{ }^{\circ} \mathrm{C}$ 时测 试和模拟的总内阻可知, 界面电阻占总内阻 $11 \%$, 界面平均电阻率为 $18.7 \mu \Omega \cdot \mathrm{cm}^{2}$ 。 $\mathrm{Li}$ 等 ${ }^{[43]}$ 研究了银、 铜䈃直接连接 $\mathrm{PbTe}$ 材料, 采用 $550{ }^{\circ} \mathrm{C}$ 热压 $3 \mathrm{~h}$, 老 化试验表明 $\mathrm{Cu}$ 比 $\mathrm{Ag}$ 元素扩散更剧烈, 在 $\mathrm{PbTe}$ 基 体内都生成了 $\mathrm{Cu}_{2} \mathrm{Te} 、 \mathrm{Ag}_{2} \mathrm{Te}$ 分散相，因此银、铜不 适合作为接触材料。

\section{3 填充方钴矿热电材料的界面}

填充方钴矿材料(Skutterudite, SKD)组分比较复 杂, $\mathrm{n}$ 型材料一般为 $\mathrm{M}_{x} \mathrm{Co}_{4} \mathrm{Sb}_{12}, \mathrm{p}$ 型材料一般为 $\mathrm{N}_{y} \mathrm{Fe}_{4-z} \mathrm{Co}_{z} \mathrm{Sb}_{12}(z<2), \mathrm{M} 、 \mathrm{~N}$ 为一种或多种填充元素。 Garcia-Canadas 等 ${ }^{[44]}$ 采用 $\mathrm{Zn}_{78} \mathrm{Al}_{22}$ 真空钎焊连接镀 $\mathrm{Pd}$ 的 SKD 和 $\mathrm{Cu}$ 电极, 通过测量器件在空气中发电 时输出功率参数, 推导出接触电阻率平均值 $477 \mu \Omega \cdot \mathrm{cm}^{2}$, 热循环 7 次后器件内阻由 $0.7 \Omega$ 变为 $8 \Omega$, 恶化主要是由于材料的氧化。Ni 以其良好的耐 氧化性和焊接性能, 也可作为 SKD 的接触材料; 但 $\mathrm{Ni}$ 的热膨胀系数 $13 \times 10^{-6} \sim 17 \times 10^{-6} \mathrm{~K}^{-1}$ 与 $\mathrm{n}$ 型 $\mathrm{SKD}$ 的热膨胀系数 $9.5 \times 10^{-6} \sim 10.5 \times 10^{-6} \mathrm{~K}^{-1}$ 相差较大 ${ }^{[45]}$ 。 此外, 由于 $\mathrm{Sb}$ 的扩散, $\mathrm{Ni}$ 高温下可与 SKD 形成化 合物。为此, 需要设计缓冲层和阻挡层来降低 $\mathrm{Ni}$ 、 SKD 间的热应力并抑制元素扩散。

$\mathrm{Mo}$ 或 $\mathrm{Mo}-\mathrm{Cu}$ 合金也可用作 SKD 的接触材料, 可以通过改变 $\mathrm{Mo} / \mathrm{Cu}$ 比例调节热膨胀系数。 Salvador 等 ${ }^{[46]}$ 采用电弧喷涂法形成 SKD 的 Mo 金属 化层, 制造了 32 对偶臂的热电器件。但是, Mo 或 $\mathrm{Mo}-\mathrm{Cu}$ 很难与 SKD 直接键合, 需要引入 $\mathrm{Ti}$ 粘附层 ${ }^{[47]}$, $\mathrm{Ti}$ 的热膨胀系数为 $8.6 \times 10^{-6} \mathrm{~K}^{-1}$, 比较接近 $\mathrm{CoSb}_{3}$ 的热膨胀系数。Zhao 等 ${ }^{[48-49]}$ 采用 $\mathrm{Ti}$ 粉作为缓冲层, 利用放电等离子体共烧结 $\mathrm{n}$ 型 SKD 和 $\mathrm{Mo}-\mathrm{Cu}$ 或 $\mathrm{W}-\mathrm{Cu}$ 合金电极，获得的总界面电阻率为 $20 \sim$ $30 \mu \Omega \cdot \mathrm{cm}^{2}$ 和结合强度为 $50 \mathrm{MPa}$, 在 $\mathrm{CoSb}_{3} / \mathrm{Ti}$ 界面 形成了 $\mathrm{TiSb} 、 \mathrm{TiSb}_{2} 、 \mathrm{TiCoSb}$ 合金。为降低 $\mathrm{Ti}$ 和 n-SKD 的界面反应以及接触电阻，加入 $6 \% \sim 15 \% \mathrm{Al}$ 粉，以 $\mathrm{TiAl}$ 合金取代 $\mathrm{Ti}$ ，两者初始接触电阻率都为 $10 \mu \Omega \cdot \mathrm{cm}^{2}$, 但 $600{ }^{\circ} \mathrm{C}$ 老化试验 $16 \mathrm{~d}$ 后, 前者电阻率 仍为 $12 \mu \Omega \cdot \mathrm{cm}^{2}$, 而后者升至 $53 \mu \Omega \cdot \mathrm{cm}^{2}$, 并且前者 的结合强度更好 ${ }^{[50]}$ 。Fan 等 ${ }^{[45]}$ 以 $\mathrm{Ti}-\mathrm{Mo} / \mathrm{Mo}-\mathrm{Cu}$ 为阻 挡缓冲复合层抑制扩散和降低 $\mathrm{Yb}_{0.3} \mathrm{Co}_{4} \mathrm{Sb}_{12}$ 与 $\mathrm{Ni}$ 的 热应力, 发现 $\mathrm{Mo}_{55} \mathrm{Cu}_{45}$ 具有最优的应力缓冲作用, $\mathrm{Ti}_{95} \mathrm{Mo}_{5}$ 具有最优的阻挡作用，其接触电阻率初始 
值 $3 \mu \Omega \cdot \mathrm{cm}^{2}, 550{ }^{\circ} \mathrm{C}$ 陈化 $12 \mathrm{~d}$ 后接触电阻率保持在 $9 \mu \Omega \cdot \mathrm{cm}^{2}$ 。Tang 等 ${ }^{[51]}$ 设计了 $\mathrm{Yb}_{0.3} \mathrm{Co}_{4} \mathrm{Sb}_{12} / \mathrm{Ti}_{0.88} \mathrm{Al}_{0.12} /$ $\mathrm{Ni}$ 热电臂结构和 $\mathrm{Ag}-\mathrm{Cu}-\mathrm{Zn} / \mathrm{Mo}-\mathrm{Cu}$ 钎焊工艺, 两者 接触电阻率初始值 $6.1 \mu \Omega \cdot \mathrm{cm}^{2}, 500{ }^{\circ} \mathrm{C}$ 陈化 $30 \mathrm{~d}$ 接触 电阻率保持在 $9.8 \mu \Omega \cdot \mathrm{cm}^{2}$ 。

$\mathrm{Gu}$ 等 ${ }^{[52]}$ 比较了 $\mathrm{p}$ 型和 $\mathrm{n}$ 型 $\mathrm{SKD}$ 与 $\mathrm{Ti}$ 的连接界 面, 发现 $\mathrm{p}$ 型 $\mathrm{Ce}_{y} \mathrm{Fe}_{x} \mathrm{Co}_{4-x} \mathrm{Sb}_{12}$ 与 $\mathrm{Ti}$ 的反应层较薄, 界 面电阻率更低, 初始值为 $3 \mu \Omega \cdot \mathrm{cm}^{2}, 550{ }^{\circ} \mathrm{C}$ 陈化 $30 \mathrm{~d}$ 后仍小于 $6 \mu \Omega \cdot \mathrm{cm}^{2}$ 。C aillat 等 ${ }^{[33]}$ 采用热压法制备了 SKD 热电偶臂, 测量得到 $\mathrm{p}$ 型 $\mathrm{Ce}_{0.85} \mathrm{Fe}_{3.5} \mathrm{Co}_{0.5} \mathrm{Sb}_{12}$ 与 $\mathrm{Ti}$ 的接触电阻率初始值也小于 $5 \mu \Omega \cdot \mathrm{cm}^{2}$ 。2012 年 Fleurial 等 ${ }^{[54]}$ 制作了 $\mathrm{p}$ 型、 $\mathrm{n}$ 型方钴矿的 $\mathrm{CoSb}_{3} / \mathrm{Zr} / \mathrm{Ti}$ 接触结构, $\mathrm{Zr}$ 作为阻挡层, 电阻率达到 $19 \mu \Omega \cdot \mathrm{cm}^{2}$; 热老化测试后, $\mathrm{CoSb}_{3} / \mathrm{Zr}$ 界面同样出现了化合物中 间层。Guo 等 ${ }^{[55]}$ 采用电弧熔法制备了 $\mathrm{Co}-\mathrm{Fe}-\mathrm{Ni}$ 基合 金，优化了热膨胀系数，并作为 $\mathrm{p}$ 型、 $\mathrm{n}$ 型 SKD 的 电极材料和阻挡层材料, 测试了方钴矿器件发电功 率和效率, 热老化试验 100 次后器件性能依然稳定。 Muto 等 ${ }^{[56]}$ 采用 $\mathrm{Co}_{2} \mathrm{Si} 、 \mathrm{CoSi}_{2}$ 作为 $\mathrm{p}$ 型、 $\mathrm{n}$ 型的接触 材料, 接触电阻率低至 $1 \sim 2 \mu \Omega \cdot \mathrm{cm}^{2}$, 但硅化物熔点 高, 需要在 $1200{ }^{\circ} \mathrm{C}$ 单独烧结制备。最近, Zhang 等 ${ }^{[13]}$ 制备得到 $12 \%$ 热电转换效率的 $\mathrm{Bi}_{2} \mathrm{Te}_{3} / \mathrm{SKD}$ 两段式 器件, 展现出 SKD 用于制造中温型热电器件的良好
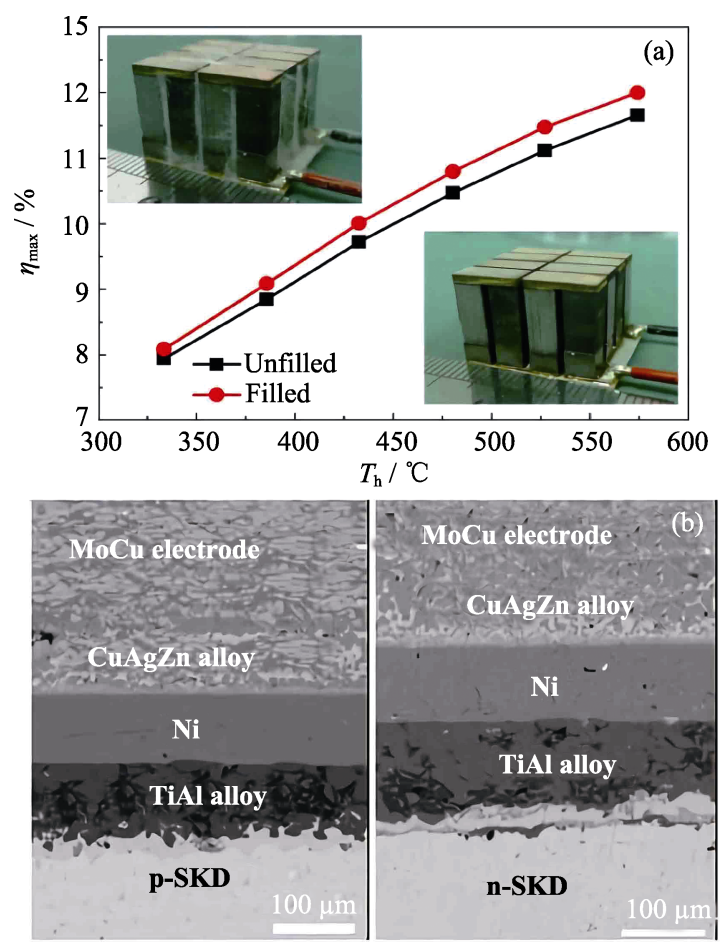

图 9 碲化铋-方钴矿两段式热电器件的发电效率(a)和器件 热端 $\mathrm{SKD} / \mathrm{Ti}_{0.88} \mathrm{Al}_{0.12} / \mathrm{Ni}$ 界面与电极扫描电镜照片(b) ${ }^{[13]}$ Fig. 9 (a) Power generation efficiency of segmented BT/SKD modules and (b) scanning electron microscopy image of $\mathrm{SKD} / \mathrm{Ti}_{0.88} \mathrm{Al}_{0.12} / \mathrm{Ni}$ interface and electrode on hot side ${ }^{[13]}$
前景。他们设计了 $\mathrm{CoSb}_{3} / \mathrm{Ti}_{0.88} \mathrm{Al}_{0.12} / \mathrm{Ni}$ 热端接触结 构, 并使用 $\mathrm{Cu}-\mathrm{Ag}-\mathrm{Zn}$ 合金钎焊到 $\mathrm{Mo}_{0.5} \mathrm{Cu}_{0.5}$ 电极, 如图 9 所示, 其中 $\mathrm{Ni}$ 作为接触层, $\mathrm{Ti}_{0.88} \mathrm{Al}_{0.12}$ 为阻挡 层, 初始的和热老化后的 $\mathrm{SKD} / \mathrm{Ti}_{0.88} \mathrm{Al}_{0.12} / \mathrm{Ni}$ 界面电 阻率均小于 $10 \mu \Omega \cdot \mathrm{cm}^{2}$ 。

\section{5 结束语}

为真正发挥热电温差发电技术在节能减排、可 靠电源和分布式、低质量热能利用方面的产业化应 用, 半导体热电技术的科技工作者们在获得高性能 热电材料的同时, 需要从器件应用角度进一步考查 其可行性。其中的一项关键技术就是热电材料和金 属电极之间低电阻/高强度和高温稳定的界面连接, 涉及到界面设计、界面材料、制造工艺以及相关评 价测试标准。

结合上述的热电转换器件失效实例和已有经验, 热电发电器件界面材料的设计至少需要考虑: 热膨 胀系数与热电材料匹配; 界面具有高温稳定性; 结 合强度大, 焊接性能好; 界面接触电阻低, 自身扩 散或反应产物不会增加接触电阻; 能够抑制焊料元 素扩散等。

单一材料往往不能同时满足以上要求, 界面材 料可能还包括扩散阻挡层, 应力缓冲层和焊接粘附 层。因此, 研制热电器件要求开发出与热电材料匹 配的界面材料, 建立系统的热电界面材料体系。期 待有更多热电材料领域的同行能够关注热电界面材 料, 加速高性能热电器件的研发, 让热电能源转换 技术能够在航空航天、新能源、光通信等众多领域 获得广泛应用。

\section{参考文献:}

[1] 中国建筑节能协会. 中国建筑能耗研究报告(2017 年), 上海, 2017.

[2] 陈立东, 刘睿恒, 史迅. 热电材料与器件. 北京: 科学出版社, 2018: 1-14.

[3] LIU W S, HU J Z, ZHANG S M, et al. New trends, strategies and opportunities in thermoelectric materials: a perspective. Mater. Today Phys., 2017, 1: 50-60.

[4] ZHU T J, LIU Y T, FU C G, et al. Compromise and synergy in high efficiency thermoelectric materials. Adv. Mater, 2017, 29(14): 1605884.

[5] LI J F, LIU W S, ZHAO L D, et al. High-performance nanostructured thermoelectric materials. NPG Asia Mater, 2010, 2(4): 152-158.

[6] ZEBARJADI M, ESFARJANI K, DRESSELHAUS M S, et al. Perspectives on thermoelectrics: from fundamentals to device applications. Energy Environ. Sci., 2012, 5(1): 5147-5162.

[7] CHEN L D, XIONG Z, BAI S Q. Recent progress of thermoelectric nano-composites. Journal of Inorganic Materials, 2010, 25(6): 561-568. 
[8] ZHAN B, LAN J Z, LIU Y C, et al. Research progress of oxides thermoelectric materials. Journal of Inorganic Materials, 2014, 29(3): 237-244.

[9] CHEN G, LIU T X, TANG X F, et al. Optimization of electrode material and connecting process for $\mathrm{Mg}-\mathrm{Si}-\mathrm{Sn}$ based thermoelectric device. Journal of Inorganic Materials, 2015, 30(6): 639-646.

[10] FU C, BAI S, LIU Y, et al. Realizing high figure of merit in heavy band p-type half-Heusler thermoelectric materials. Nat. Comm., 2015, 6: 8144-8151.

[11] HU X, JOOD P, OHTA M, et al. Power genaration of nanostructured PbTe-based thermoelectrics: comprehensive development from materials to modules. Energy Environ. Sci., 2016, 9(2): $517-529$.

[12] KRAEMER D, JIE Q, MCENANEY K, et al. Concentrating solar thermoelectric generator with a peak efficiency of $7.4 \%$. Nature Energy, 2016, 1: 1-8.

[13] ZHANG Q, LIAO J, TANG Y, et al. Realizing a thermoelectric conversion efficiency of $12 \%$ in bismuth telluride/skutterudite segmented modules through full-parameter optimization and energyloss minimized integration. Energy Environ. Sci., 2017, 10(4): 956-963.

[14] HAO F, QIU P, TANG Y, et al. High efficiency $\mathrm{Bi}_{2} \mathrm{Te}_{3}$-based materials and devices for thermoelectric power generation between 100 and $300^{\circ}$ C. Energy Environ. Sci., 2016, 9(10): 3120-3127.

[15] 张建中. 温差电技术. 天津: 天津科学技术出版社, 2013: 131-135, 219-224.

[16] 张文典. 实用表面组装技术, 4 版. 北京: 电子工业出版社, 2015: 162-247.

[17] HATZIKRANIOTIS E, ZORBAS K T, SAMARAS I, et al. Efficiency study of a commercial thermoelectric power generator (TEG) under thermal cycling. J. Electron. Mater., 2010, 39(9): 2112-2116.

[18] PARK W, BARAKO M T, MARCONNET A M, et al. Effect of thermal cycling on commercial thermoelectric modules. 13th Intersociety Conference on Thermal and Thermomechanical Phenomena in Electronic Systems, San Diego, 2012, 16(12): 107-112.

[19] CLIN TH, TURENNE S, VASILEVSKIY D, et al. Numerical simulation of the thermomechanical behavior of extruded bismuth telluride alloy module. J. Electro. Mater., 2009, 38(7): 994-1001.

[20] KIM H S, WANG T, LIU W S, et al. Engineering thermal conductivity for balancing between reliability and performance of bulk thermoelectric generators. Adv. Funct. Mater., 2016, 26(21): 3678-3686.

[21] LIU W S, WANG H, WANG L, et al. Understanding of the contact of nanostructured thermoelectric n-type $\mathrm{Bi}_{2} \mathrm{Te}_{2.7} \mathrm{Se}_{0.3}$ legs for power generation applications. J. Mater. Chem. A, 2013, 1(42): 13093-13100.

[22] LAN Y C, WANG D Z, CHEN G, REN Z F. Diffusion of nickel and tin in p-type $(\mathrm{Bi}, \mathrm{Sb})_{2} \mathrm{Te}_{3}$ and n-type $\mathrm{Bi}_{2}(\mathrm{Te}, \mathrm{Se})_{3}$ thermoelectric materials. Appl. Phys. Lett., 2008, 92(10): 101910-1-3.

[23] ROWE D M. CRC Handbook of Thermoelectrics. USA: CRC Press LLC, 1995: 479-485.

[24] LIU W S, QING J, KIM H S, et al. Current progress and future challenges in thermoelectric power generation: from materials to devices. Acta Materialia, 2015, 87: 357-376.

[25] HABA V. Method and Materials For Obtaining Low Resistance Bond to Bismuth Telluride. US Patent, 3017693, 1962. US Patent, $3079455,1963$.

[26] ROSI F D, BERNOFF R A. Method and Materials for Obtaining Low Resistance Bonds to Thermoelectric Bodies. US Patent, 3037064, 1962.

[27] LIAO C N, LEE C H, CHEN W J. Effect of interfacial compound formation on contact resistivity of soldered junction between bis- muth telluride based thermoelements and copper. Electrochem. Solid-State Lett., 2007, 10(9): 23-25.

[28] MENGALI O J, SEILER M R. Contact resistance studies on thermoelectric materials. Adv. Energy Conversion, 1962, 2(62): 59-68.

[29] WEITZMAN L H. Etching Bismuth Telluride. US Patent, 3338765 , 1967.

[30] TALOR P J, MADDUX J R, MEISSNER G, et al. Controlled improvement in specific contact resistivity for thermoelectric materials by ion implantation. Appl. Phys. Lett., 2013, 103(4): 043902-1-4.

[31] LIN W P, WESOLOWSKI D E, LEE C C. Barrier/bonding layers on bismuth telluride for high temperature thermoelectric modules. J. Mater. Sci.: Mater. Electron., 2011, 22(9): 1313-1320.

[32] IYORE O D. Interface Characterization of Contacts to Bulk Bismuth Telluride Alloys. Richardson, TX: University of Texas at Dallas, Master's Thesis, UMI No. 1470835, 2009.

[33] FENG H P, YU B, CHEN S, et al. Studies on surface preparation and smoothness of nanostructured $\mathrm{Bi}_{2} \mathrm{Te}_{3}$-based alloys by electrochemical and mechanical methods. Electrochimica Acta, 2011, 56(8): 3079-3084.

[34] IYORE O D, LEE T H, GUPTA R P, et al. Interface characterization of nickel contact to bulk bismuth telluride selenide. Surf. Interface Analysis, 2009, 41(5): 440-444.

[35] WEINSTEIN M, MLAVSKY A I. Bonding of lead telluride to pure iron electrodes. Rev. Sci. Instrum., 1962, 33(10): 1119-1120.

[36] SINGH A, BHATTACHARYA S, THINAHARAN C, et al. Development of low resistance electrical contacts for thermoelectric devices based on n-type PbTe and p-type TAGS-85 $\left(\left(\mathrm{AgSbTe}_{2}\right)_{0.15}(\mathrm{GeTe})_{0.85}\right)$. J. Phys. D: Appl. Phys., 2008, 42(1): 015502-1-6.

[37] LEAVITT F A, MCCOY J W, MARUDHACHALAM P, et al. Segmented Thermoelectric Module with Bonded Legs. US Patent, 2012/0103381 A1, 2012.

[38] XIA H, DRYMIOTIS F, CHEN C L, et al. Bonding and high-temperature reliability of NiFeMo alloy/n-type $\mathrm{PbTe}$ joints for thermoelectric module applications. J. Mater. Sci., 2015, 50(7): 2700-2708.

[39] XIA H, DRYMIOTIS F, CHEN C L, et al. Bonding and interfacial reaction between $\mathrm{Ni}$ foil and $\mathrm{n}$-type $\mathrm{PbTe}$ thermoelectric materials for thermoelectric module applications. J. Mater. Sci., 2014, 49(4): 1716-1723.

[40] XIA H, CHEN C L, DRYMIOTIS F, et al. Interfacial reaction between $\mathrm{Nb}$ foil and n-type PbTe thermoelectric materials during thermoelectric contact fabrication. J. Electro. Mater., 2014, 43(11): 4064-4069.

[41] ORIHASHI M, NODA Y, CHEN L, et al. Ni/n-PbTe and $\mathrm{Ni} / \mathrm{p}-\mathrm{Pb}_{0.5} \mathrm{Sn}_{0.5}$ Te Joining by Plasma Activated Sintering. 17th International Conference on Thermoelectrics, Nagoya, 1998: 543-546.

[42] FERRERES X R, YAMINI S A, NANCARROW M, et al. One-step bonding of Ni electrode to n-type PbTe - a step towards fabrication of thermoelectric generators. Materials and Design, 2016, 107: 90-97.

[43] LI C C, DRYMIOTIS F, LIAO L L, et al. Interfacial reactions between $\mathrm{PbTe}$-based thermoelectric materials and $\mathrm{Cu}$ and $\mathrm{Ag}$ bonding materials. J. Mater. Chem. C, 2015, 3(40): 10590-10596.

[44] GARCIA-CANADAS J, POWELL A V, KALTZOGLOU A, et al. Fabrication and evaluation of a skutterudite-based thermoelectric module for high-temperature applications. J. Electro. Mater, 2013, 42(7): 1369-1374.

[45] FAN X C, GU M, SHI X, et al. Fabrication and reliability evaluation of $\mathrm{Yb}_{0.3} \mathrm{Co}_{4} \mathrm{Sb}_{12} / \mathrm{Mo}-\mathrm{Ti} / \mathrm{Mo}-\mathrm{Cu} / \mathrm{Ni}$ thermoelectric joints. $\mathrm{Ce}$ ramics International, 2015, 41(6): 7590-7595.

[46] SALVADOR J R, CHO J Y, YE Z, et al. Conversion efficiency of 
skutterudite-based thermoelectric modules. Phys. Chem. Chem. Phys., 2014, 16(24): 12510-12520.

[47] FAN J F, CHEN L D, BAI S Q, et al. Joining of Mo to $\mathrm{CoSb}_{3}$ by spark plasma sintering by inserting a Ti interlayer. Materials Letters, 2004, 58(30): 3876-3878.

[48] ZHAO D G, GENG H R, TENG X Y. Fabrication and reliability evaluation of $\mathrm{CoSb}_{3} / \mathrm{W}-\mathrm{Cu}$ thermoelectric element. J. Alloys Compd., 2012, 517(7): 198-203.

[49] ZHAO D G, LI X Y, JIANG W, et al. Fabrication of $\mathrm{CoSb}_{3} / \mathrm{MoCu}$ thermoelectric joint by one-step SPS and evaluation. Journal of Inorganic Materials, 2009, 24(3): 545-548.

[50] GU M, XIA X G, LI X Y, et al. Microstructural evolution of the interfacial layer in the $\mathrm{Ti}-\mathrm{Al} / \mathrm{Yb}_{0.6} \mathrm{Co}_{4} \mathrm{Sb}_{12}$ thermoelectric joints at high temperature. J. Alloys Compd., 2014, 610: 665-670.

[51] TANG Y S, BAI S Q, REN D D, et al. Interface structure and electrical property of $\mathrm{Yb}_{0.3} \mathrm{Co}_{4} \mathrm{Sb}_{12} / \mathrm{Mo}-\mathrm{Cu}$ element prepared by welding using $\mathrm{Ag}-\mathrm{Cu}-\mathrm{Zn}$ solder. Journal of Inorganic Materials,
2015, 30(3): 256-260.

[52] GU M, XIA X G, HUANG X Y, BAI S Q, et al. Study on the interfacial stability of p-type $\mathrm{Ti} / \mathrm{Ce}_{y} \mathrm{Fe}_{x} \mathrm{Co}_{4-x} \mathrm{Sb}_{12}$ thermoelectric joints at high temperature. J. Alloys Compd., 2016, 671: 238-244.

[53] CAILlAT T, FLEURIAL J P, SNYDER G J, et al. Development of High Efficiency Segmented Thermoelectric Unicouples. Proceedings of 20th Int. Conf. on Thermoelectrics, Beijing, 2001, 504(1): 282-285.

[54] FLEURIAL J P, CAILLAT T, CHI S C. Electrical Contacts for Skutterudite Thermoelectric Materials. US Patent, 20120006376 A1, 2012.

[55] GUO J Q, GENG H Y, OCHI T, et al. Development of skutterudite thermoelectric materials and modules. J. Electro. Mater., 2012, 41(6): 1036-1042.

[56] MUTO A, YANG J, POUDEL B, et al. Skutterudite unicouple characterization for energy harvesting applications. Adv. Energy Mater, 2013, 3(2): 245-251. 